\title{
Experimental realization of multi-service RoF system using OCS-PolMux techniques
}

\begin{abstract}
Optical Carrier Suppression has been proven as an efficient way of generating high frequency optical carrier for Radio over Fiber (RoF) systems. However, previous studies show that OCS with the combination of RF and optical components can only transmit a single service in RoF system. This paper shows that two different services can be transmitted using a single wavelength through RoF system with the use of Polarization Multiplexing (PolMux) method. The combination of these two techniques enables multiservice RoF such as LTE and WiFi services to be transmitted simultaneously to the customers.
\end{abstract}

Keyword: Local Multipoint Distribution Service (LMDS); Multiservice; Optical Carrier Suppression (OCS); Polarization Multiplexing (PolMux); Radio over Fiber (RoF) 